\title{
ASPECTOS CORPORALES Y SOCIALES A TENER EN CUENTA EN UNA EDUCACIÓN INCLUSIVA
}

\author{
ASPECTOS CORPORAIS E SOCIAIS A SEREM CONSIDERADOS NUMA \\ EDUCAÇÃO INCLUSIVA
}

BODILY AND SOCIAL ASPECTS TO BE CONSIDERED IN INCLUSIVE

EDUCATION

Juan Bautista Llorens Gómez*, Ana María Díaz Olaya*

\begin{abstract}
Palabras clave:
Educación.

Movimiento.

Cuerpo humano.

Niño.

Resumen: La danza integrada, dentro de las artes escénicas inclusivas, encierra una serie de singularidades que son extrapolables a la educación. Esta experiencia es una investigación-acción en la que se pretende conocer cuáles son las herramientas que utiliza el profesorado de Educación Infantil para optimizar la convivencia en el aula, así como su relación con los aspectos corporales y sociales de una educación inclusiva. Las conclusiones se han extraído de la realización de grupos de discusión con profesores en cuatro colegios públicos andaluces. Paralelamente, se ha diseñado un programa de intervención del que se incluye una de las unidades didácticas; en ella se puede observar cómo se plasman en un ámbito educativo los aspectos citados.
\end{abstract}

Palavras chave:

Educação.

Movimento.

Corpo humano.

Criança.

\section{Keywords:}

Education.

Motion.

Human body.

Child.

Resumo: A dança integrada, dentro das artes cênicas inclusivas, contém uma série de singularidades que podem ser extrapoladas para a educação. Esta experiência é uma pesquisa-ação que se destina a saber quais são as ferramentas utilizadas pelos professores da educação infantil para otimizar a convivência na sala de aula, bem como a relação destes com os aspectos corporais e sociais da uma educação inclusiva. As conclusões foram extraídas de grupos de discussão com professores em quatro escolas públicas da Andaluzia. Paralelamente, foi projetado um programa de intervenção, incluindo uma das unidades de ensino, na qual é possível observar como os aspectos mencionados se refletem num ambiente educacional.

Abstract: Within Inclusive Performing Arts, integrated dance encompasses a series of singularities which can be transposed to Education. This experience is an action-research study aimed at finding the tools applied by teachers in Early Childhood Education to optimize school life as well as the relationship between these tools and inclusive education's bodily and social aspects. The findings are derived from discussion groups in four state schools in Andalusia. In parallel, an intervention program has been designed, including an educational unit where we can observe how bodily and social aspects are reflected in an educational environment.
*Universidad de Málaga. Málaga,
España. E-mail: jbllorens@ @uma.es; España. E-mail: jbllorer

Recebido em: 05/05/2018 Aprovado em: 07/11/2018

DOl: https://doi.org/10.22456/1982-8918.82614 (c) (i) () Licence 


\section{INTRODUCCIÓN}

Descartes culminó el proceso de separación cuerpo/mente dando protagonismo a esta última, la razón. Quedaba así el cuerpo subordinado a un segundo lugar, y por lo tanto relegadas la concepción que de él y de sus variantes se ha tenido. Es por esto que el movimiento y la danza no han estado ni están lo suficientemente presentes en la educación; por una parte, porque las políticas educativas no han fomentado la presencia de la danza y el movimiento en el sistema educativo español (ORTIZ, 2015), y por otra parte, por la insuficiente formación del profesorado en estas materias (LAGO; ESPEJO, 2007; MONTOYA, 2015; ORTIZ, 2015).

Esta dicotomía cuerpo/mente también se refleja en los sentidos tacto/visión (PALLASMAA, 2012), y como consecuencia la preponderancia de lo visual sobre lo táctil en las aulas, y de lo individual sobre lo comunitario (CASANOVA, 1991; KIRK, 1989).

Si estas observaciones se contextualizan en un entorno inclusivo, que es el paradigma por el que apuesta esta investigación, se hablaría siempre de educación inclusiva (BOOTH; AINSCOW, 2015; FERNÁNDEZ, 2005/06; HOPKINS; AINSCOW; WEST, 1999; MORIÑA, 2004; PIJL; MEIJER; HEGARTY, 1997) y de danza integrada (BENJAMIN, 2012; BRUGAROLAS, 2016); y es en este contexto donde surge el objetivo principal de esta investigación: entender y comprender en profundidad cuáles son las preocupaciones y las estrategias educativas del profesorado de educación infantil en el aula en cuanto a la convivencia escolar, relacionando estas con los aspectos corporales y sociales de una educación inclusiva, como respuesta a una tendencia a lo visual y al individualismo en los procesos educativos y en la sociedad en general.

Las experiencias que han posibilitado esta investigación se deben, en primer lugar, a la participación en el proyecto Educación Musical Infantil que la Fundación Pública Andaluza Barenboim-Said (FPABS), bajo convenio con el gobierno autonómico, tiene en colegios públicos de Andalucía ${ }^{1}$-de los cuales se han seleccionado para este estudio cuatro colegios andalucesy en segundo lugar, a la experiencia con la danza integrada a través de los últimos cinco años de trayectoria artística del Colectivo La Tralla. Además, la investigación se acompaña de un programa de intervención que está compuesto por diez unidades didácticas y que se han diseñado para ser puestas en práctica en los citados centros educativos durante veinte sesiones. Las unidades están basadas en los aspectos corporales y sociales de una educación inclusiva: sentido del tacto, exploración y comunitarismo (véase epígrafe 2). Hay que aclarar que estos derivan del análisis de las singularidades de la danza integrada y de su trasposición al campo educativo.

Estos tres aspectos/singularidades son la base del marco teórico tanto de la investigación-acción como del propio programa de intervención, y surgen de la profundización en varias ciencias sociales. De la antropología/sociología se obtiene el aspecto comunitario (GOBERNADO, 1986, 1990; KRAUSE, 2001; LE BRETON, 2003; NOVACK, 1990; SPENCER, 1985; TAMPINI, 2012), de la filosofía/sociología el exploratorio (DELEUZE; GUATARI, 2004; FOUCAULT, 2012; GARCÉS, 2013; KUPPERS, 2000; MERLEAU-PONTY, 1993; TAMPINI, 2012), y de la historia de la danza el sentido del tacto (BANES, 1987; BENJAMIN, 2002; BROZAS, 2017; BRUGAROLAS, 2015; HÜBNER, 2005; y NOVACK, 1990).

1 La investigación cumple con la normativa vigente en relación con los derechos y bienestar de las personas implicadas en la investigación y posee el informe del CEUMA (Comité Ético de Experimentación de la Universidad de Málaga), además de encontrarse el proyecto docente 
Por otra parte se han ampliado y complementado los argumentos anteriores desde el ámbito educativo (BERNSTEIN, 1990; CASANOVA, 1991; FREINET, 1963; FROEBEL, 2003; KEY, 1906; KIRK, 1989; MAKARENKO, 1977; MONTESSORI, 2003), la psicología del desarrollo (FERNÁNDEZ; MELERO, 1995; TUDGE; ROGOFF, 1995) y la neurociencia educativa (GOLDING, 2016; ROMERO, 2016).

En relación a otras experiencias que pudieran surtir de conocimiento a esta investigación en los campos de la danza, la educación y los tres aspectos corporales y sociales descritos, se han encontrado las siguientes aportaciones: desde Froebel (2003) en el siglo XVIII/XIX se viene destacando la importancia del tacto en el desarrollo del alumnado (GIMENO, 1986; MONTESSORI, 2003), y es en este campo donde Henley (2014) utiliza los sentidos para fomentar el carácter crítico del discente a través de un educador de la danza. Por su parte, Kirk (1989) y Casanova (1991) han puesto el acento en el aspecto cooperativo de la educación, como lo hicieran anteriormente Freinet (1972) o Makarenko (1977). A este respecto, Russell (1965) traslada este acento a la simbiosis danza/comunitarismo. Por último, en el ámbito exploratorio, Key (1906) y Froebel (2003) realizan interesantes aportaciones. Sin embargo, al extrapolarlo al campo del movimiento, no se han encontrado experiencias que le otorguen protagonismo, aunque sí formando parte de las mismas en un entorno que acentúa la estimulación cognitiva (GOLDING, 2016; ROMERO, 2012).

Todas estas investigaciones están en relación a esta por el contexto educativo y dancístico. Sin embargo, no se han encontrado estudios para poder confrontarla en relación a los tres aspectos corporales y sociales citados. Aquí se muestran estrategias que enfatizan lo corporal en relación a la convivencia escolar en un contexto inclusivo, mientras que las publicaciones encontradas acentúan los aspectos contrarios -sentido de la vista, enseñanza directiva e individualismo- a través de la tecnología, los medios audiovisuales y otras estrategias de enseñanza (CABERO, 2015; PÉREZ GÓMEZ, 2012), como se verá en detalle en la discusión y en la conclusión final.

Por último, aclarar que este artículo da respuesta al objetivo planteado, y aunque no ofrece conclusiones referentes al programa de intervención, sí lo acompaña de una unidad didáctica del citado programa inspirada en un tema popular brasileño, Samba lelé (véanse anexos 1 y 2). Con ella se muestra cómo llevar al aula los aspectos corporales y sociales de una educación inclusiva que se exponen a continuación.

\section{EDUCACIÓN INCLUSIVA Y DANZA INTEGRADA}

Se procede entonces a esclarecer el germen de las tres singularidades de la danza integrada y su uso como aspectos corporales y sociales en una educación inclusiva: sentido del tacto, exploración y comunitarismo.

El sentido del tacto en la historia de la danza es el relato de una reconciliación que nace con la danza moderna a principios del siglo XX en Europa, de la mano de Rudolf von Laban, y termina con una de la manifestaciones de la danza posmoderna en la década de los setenta, el Contact Improvisation (CI) (LLORENS, 2017). Creado por Steve Paxton, su característica más relevante es su lectura como una puesta en escena no verbal de las revoluciones sociales que se estaban dando en la época (BANES, 1987). Esto se manifiesta al enumerar sus principales características: ausencia de coreógrafo, desaparición de roles de género, exploración de los 
sentidos, improvisación y participación generalizada (NOVACK, 1990). El Cl procuraría en breve la herramienta más adecuada para el trabajo de la danza integrada, y esto se debe al alto valor inclusivo de dos de sus cualidades: la improvisación y el tacto (BENJAMIN, 2002; BRUGAROLAS, 2015; BROZAS, 2017; HÜBNER, 2005).

Al mismo tiempo que en la danza, el sentido del tacto también recupera su protagonismo en el ámbito educativo. A finales del XIX comienzan una serie de movimientos de renovación pedagógica que tienen en común su apuesta por los sentidos (FREINET, 1972; KEY, 1906; LUZURIAGA, 1923; MADRID; MAYORGA, 2012; MAKARENKO, 1977; MONTESSORI, 2003).

El comunitarismo, cuyo concepto se puede ver con detalle en Gobernado $(1986,1999)$, es una invitación antropológico/sociológica a la mediación con formas comunitarias ancestrales (LLORENS, 2017) existentes con anterioridad al individualismo. Este último se fue imponiendo en la sociedad desde el trescento italiano, donde los intereses propios comenzaban a primar sobre los de la comunidad (LE BRETON, 1990). Esto contrasta con una concepción corporal menos individualista y opuesta que se conserva con un carácter inclusivo en las tradiciones populares (LE BRETON, 1990).

En la misma línea pero en el entorno educativo encontramos defensores del fomento del comunitarismo en la escuela. El curriculum básico puede ser el medio mediante el cual impulsar el valor social de la educación en una sociedad individualista (CASANOVA, 1991; KIRK, 1989). O bien el cuerpo una forma de entender y controlar los procesos de socialización en espacios escolares (MARTINELLI; DAOLIO; FILGUEIRA, 2017).

La exploración es la última de las singularidades de la danza integrada. Llorens (2017), tomando como base las teorías de Foucault (2012), define la exploración como un acto de rebelión contra los sistemas establecidos por el poder. Con base en estas ideas filosófico/ sociológicas, y enriqueciéndolas con las teorías de Bernstein (1990) en el campo educativo, se constata cómo la disciplina corporal y la disciplina transmitida a través de los curriculums en las escuelas han contribuido a la manipulación de las masas. Una forma de reaccionar a estas disciplinas sería fomentar un lenguaje libre, la exploración/improvisación.

Se puede hablar entonces de una disciplina corporal y de una disciplina curricular. La disciplina oculta el control de los ciudadanos por parte del poder, desde un punto de vista corporal mediante el adiestramiento del cuerpo, y desde un punto de vista lingüístico mediante los currículums escolares. El acto rebelde que va a fracturar estos mecanismos de control de masas es la vuelta a la exploración y la improvisación de nuestro cuerpo y el de los otros, no solo desde el punto de vista corporal sino como lenguaje de comunicación no verbal.

\section{METODOLOGÍA}

Una investigación-acción es una "[...] forma de investigación llevada a cabo por parte de los prácticos sobre sus propias prácticas" (KEMMIS; MCTAGGART, 1988, p.42). En este caso han intervenido los profesores de segundo ciclo de educación infantil y los especialistas en música y movimiento de la FPABS de los cuatro colegios implicados.

Para dar respuesta al objetivo de la investigación, entender y comprender en profundidad cuáles son las preocupaciones y las estrategias educativas del profesorado de educación infantil en el aula en cuanto a la convivencia escolar, relacionando estas con los aspectos 
corporales y sociales de una educación inclusiva, se requieren de técnicas de investigación que permitan un conocimiento en profundidad de las experiencias de los docentes implicados. Para ello se ha utilizado el grupo de discusión. La razón principal de esta elección se debe a que el grupo de discusión es una técnica de clara orientación fenomenológica, es decir, que refleja la experiencia tal cual la perciben o la entienden los participantes: Desde la fenomenología el "nosotros/as" construye y reconstruye al propio grupo" (SUÁREZ, 2005, p.33), o dicho de otra forma: "la interacción grupal que se produce en el propio grupo se considera fundamental para la mejor comprensión de la problemática" (SUÁREZ, 2005, p.67).

El proceso de los grupos de discusión se ha hecho siguiendo las siguientes fases (SUAREZ, 2005):

PRIMERO: Selección de los centros y participantes. Se ha utilizado la red de colegios públicos malagueños donde la FPABS tiene implantado el proyecto Educación Musical Infantil: Centro Norte, Centro Sur, Centro Este y Centro Oeste (los nombres son ficticios para preservar sus identidades). Previa cita con la dirección del centro, los cuatro colegios aceptaron la participación en la investigación, con un total de quince profesores -quince aulas- de segundo ciclo de educación infantil -cursos segundo y tercero-. Por otro lado, se informó a los cuatro especialistas de la FPABS, que también aceptaron. Se realizaron tres grupos de discusión -dos colegios coincidieron en el mismo grupo-, y estuvieron conformados por los profesores de cada centro, el profesor especialista correspondiente, un observador crítico, y un moderador.

SEGUNDO: Tiempo y lugar de la celebración. Los grupos de discusión se llevaron a cabo en los propios centros educativos en horario de tarde entre los días 9 y 22 de enero de 2018, haciéndolos coincidir con las reuniones de ciclo de los diferentes centros. En ellos se cuidó una posición de igualdad de todos los participantes en la mesa, así como una atención cercana, amable y atenta que propiciara un ambiente adecuado a la discusión.

TERCERO: Soporte de grabación. Los tres grupos de discusión se grabaron con cámara de video y grabadora de audio para su posterior transcripción, previo permiso a todos los participantes.

CUARTO: Desarrollo. Tras una breve bienvenida y agradecimiento a todos los participantes se aclararon los objetivos principales de la investigación-acción y se informó sobre la confidencialidad y privacidad de toda la información generada. Seguidamente se dio paso a la discusión formulando la pregunta: ¿Qué factores crees que influyen en la convivencia escolar de tu aula?

El grupo de discusión ha permitido la implicación del profesorado en los objetivos de la investigación de una forma participativa, abierta y compartida. Con ello se pretende que los resultados puedan verse reflejados en futuras acciones del profesor tutor, y que sea esta una vía de ampliación al resto de la comunidad educativa: "Para ello trabajar desde estrategias grupales se hace indispensable debido a la visión que aportan sobre el objeto de estudio, así como por el impacto que puede tener en las personas que participan en ellas" (SUÁREZ, 2005, p.14). 


\section{RESULTADOS}

El análisis de los datos obtenidos de las transcripciones de los tres grupos de discusión realizados en los cuatro centros escolares se ha hecho siguiendo el análisis comprensivo de Taylor y Bogdan (1986). Se parte de los llamados datos en bruto, para obtener los datos en sí, es decir, fragmentos de información que tienen sentido pleno, y de estos se obtienen las categorías. Estas se han clasificado en externas, es decir, aquellas que les vienen impuestas al profesorado bien por las circunstancias sociales del entorno o por las políticas educativas del momento, y categorías internas, aquellas que sí dependen del profesorado, y son:

Cuadro 1 - Categorías

\begin{tabular}{|c|c|c|}
\hline \multirow{12}{*}{ Categorías externas } & \multirow{5}{*}{ Familia } & Normas/valores \\
\hline & & Sobreprotección \\
\hline & & Convivencia niños/padres \\
\hline & & Apoyo de las familias \\
\hline & & Nivel socio-económico \\
\hline & \multirow{2}{*}{ Sociedad } & Individualismo/comunitarismo \\
\hline & & Tecnología \\
\hline & \multirow{5}{*}{ Centros } & Ratio/Recursos \\
\hline & & Diversidad \\
\hline & & Recursos materiales \\
\hline & & Compañeros \\
\hline & & Sistema educativo \\
\hline \multirow{11}{*}{ Categorías internas } & \multirow{8}{*}{ Recursos generales } & Crear buen clima \\
\hline & & Estabelecer normas \\
\hline & & Formación \\
\hline & & Refuerzo positivo \\
\hline & & Participación familias \\
\hline & & Exploración \\
\hline & & Crear comunidad \\
\hline & & \begin{tabular}{|l|} 
Escucha \\
\end{tabular} \\
\hline & \multirow{3}{*}{$\begin{array}{l}\text { Recursos relacionados con aspectos } \\
\text { corporales }\end{array}$} & Movimiento, expresión corporaly danza \\
\hline & & Música \\
\hline & & Técnicas de relajación \\
\hline
\end{tabular}

Fonte: Grupos de discusión

De todas las categorías expuestas son de interés en este artículo aquellas que están directamente relacionadas con los aspectos corporales y sociales (véase cuadro 1 en negrita). De las categorías externas, las derivadas de la sociedad "Individualismo/comunitarismo" y "Tecnología", y las que provienen de los centros "Diversidad" y "Recursos materiales". De las categorías internas: "Exploración", "Crear comunidad", y "Escucha" en recursos generales, y "Movimiento, expresión corporal y danza", "Música" y "Técnicas de relajación" en recursos relacionados con aspectos corporales -estas dos últimas se han incluido en esta subcategoría por la implicación corporal de ambas en esta investigación-. A continuación se exponen las síntesis de estas categorías:

\subsection{Categoría: Individualismo/comunitarismo}

Esta categoría surge al comparar entornos socio-económicos opuestos. Existe mayor egocentrismo e individualismo en el alumnado proveniente de niveles socio-económicos 
medios/altos. Se puso énfasis en la necesidad de que aprendan a relacionarse en grupo y al fomento de actividades grupales en una sociedad de tendencia individualista y competitiva.

\subsection{Categoría: Tecnología}

Se discutió sobre el efecto de la tecnología en el alumnado, en concreto de las consecuencias en la forma de relacionarse. Si antes bajaban a la calle a jugar, ahora se quedan en casa y se relacionan desde las redes sociales. Se alude a la necesidad de utilizar la tecnología y las redes sociales como herramienta educativa, darle un buen uso, y no luchar contra ella.

\subsection{Categoría: Diversidad}

La diversidad, concretamente la diversidad funcional, requiere de apoyo en el aula. Se distinguió entre diversidad funcional con y sin comportamientos disruptivos. Mientras que la diversidad con comportamientos disruptivos es negativa para la convivencia, provoca miedo y rechazo al resto del grupo, la diversidad sin comportamientos disruptivos es positiva para la convivencia en el aula, despertando en el alumnado comportamientos solidarios. Por otro lado, las familias han reaccionado de diferente manera ante la inclusión de personas con diversidad funcional en el aula. Mientras las familias de nivel socio-económico medio/alto han visto con reticencias su inclusión, las familias de nivel socio-económico medio/bajo han optado por luchar para conseguir mayor apoyo de las instituciones públicas, sin rechazar su inclusión en el aula. "Lo que no saben [las familias] es que luego puede ser más beneficioso que perjudicial" (P1, C, $\left.2,26^{\prime} 25^{\prime \prime}\right)^{2}$.

\subsection{Categoría: Recursos materiales}

Los recursos materiales que solicita el profesorado son recursos digitales, concretamente la pizarra digital. De los cuatro colegios dos de ellos tenían pizarras digitales en cada una de sus aulas, y otros dos solo tenían una pizarra digital para todo el colegio. Todos alaban la labor educativa que hacen las pizarras digitales por la posibilidad inmediata de acceso a la información, además de su uso para captar la atención y tranquilizar al grupo.

\subsection{Categoría: Exploración}

En breves ocasiones se ha hecho referencia a la exploración, por una parte como elemento para trabajar la escucha y el respeto, y por otra para aprender a enfrentarse por sí solos a situaciones reales de la sociedad.

\subsection{Categoría: Crear comunidad}

Un docente, hablando como padre/madre más que como educador, informa a las familias de la importancia de crear comunidad fuera del aula para fomentar una buena convivencia entre el alumnado.

2 Dato significativo con notación compuesta por cuatro símbolos entre paréntesis: la primera letra corresponde a la persona (en este caso profesor), la segunda al centro educativo, la tercera al número de grupo de discusión, y la cuarta al minuto de la grabación. 


\subsection{Categoría: Escucha}

Dos docentes, y también en el papel de padres/madres más que de educadores, invitan al resto de profesores a dar más espacio al alumnado, respetar sus tiempos, ponerse en el lugar de ellos. Un profesor dice que el aprendizaje por proyectos, centrado en los intereses del mismo es una buena manera de motivarlos.

\subsection{Categoría: Música}

Todas las referencias a la música describen su uso para captar la atención y la relajación del grupo.

\subsection{Categoría: Movimiento, expresión corporal y danza}

El movimiento y el cuerpo se mencionan desde dos puntos de vista diferentes: como herramienta de cohesión, y como herramienta de diversión. Sin embargo, se percibe respeto y cierto recelo al movimiento como factor desencadenante del caos, principalmente por la falta de conocimiento y/o formación en este ámbito. Algunos señalan la importancia de tener sentado al grupo, y no en movimiento, y otros comentan su incapacidad de trabajar el movimiento y el cuerpo. "Es verdad, que cuando metemos música, metemos danza, metemos baile ahí ya todo como que se suaviza. Que todo parece que se iguala. Ahí ya no hay tanto pico, de que el más listo, y a lo mejor el que le cuesta más, se iguala, los dos bailan, los dos se lo pasan bomba" (P4, A, 1, 35'54”).

\subsection{Categoría: Técnicas de relajación}

Se menciona una experiencia muy positiva con mindfulness y yoga en el aula. El resto de docentes pusieron interés en su aplicación en el aula.

\section{DISCUSIÓN}

A continuación se van a contrastar y deducir planteamientos asociados con aquellas categorías que tienen relación con los aspectos corporales y sociales de una educación inclusiva. Estos no son solo el fruto de la simplificación de los datos aportados en los grupos de discusión, o dicho de otra manera, de las opiniones mayoritarias, sino que hacen alusión a todas y cada una de las opiniones de los participantes.

A modo de síntesis y como punto de partida para desgranar los resultados de esta investigación, se establece que los profesores de segundo ciclo de educación infantil de los niveles segundo y tercero que participan en la misma no recurren a actividades que tengan implicación corporal a la hora de trabajar la convivencia u otros aspectos propios del sistema de enseñanza-aprendizaje en el aula, es decir, juegos que impliquen el cuerpo, el movimiento, la danza o la expresión corporal.

En primer lugar, y respecto a las categorías externas referentes a la sociedad, "Individualismo/comunitarismo" y "Tecnología", se extrae que el tipo de sociedad en la que vivimos promueve el individualismo y la competitividad. El uso excesivo de las tecnologías 
enfatiza esta tendencia, incitando al alumnado a no interactuar cuerpo a cuerpo, sino a hacerlo a través de redes sociales. También se alude a que la tecnología está ahí para ser utilizada y no para ser culpada, como acreditan numerosas investigaciones que potencian su uso en el aula (CABERO, 2015; PÉREZ GÓMEZ, 2012), y que implícitamente fomentan el individualismo (GOBERNADO, 1991; KIRK, 1989), y conllevan al profesorado a situarse en una encrucijada en cuanto a la definición de su propia práctica docente. El profesional de la enseñanza debe plantearse, por lo tanto, cuál es su misión en este contexto, si promover la individualidad o el comunitarismo, así como la manera y las herramientas llevarla a cabo.

Con respecto al resto de categorías externas que no dependen del profesorado, en este caso del centro, se encuentran "Diversidad" y los "Recursos materiales". El profesorado demanda, de esta última, pizarras digitales, cuyos beneficios destacan algunos autores (ROBLIZO; CÓZAR, 2015; RUIZ; HERNÁNDEZ, 2018). Estas son reclamadas como herramientas de acceso inmediato a la información, pero también para ser utilizadas para captar la atención y tranquilizar al grupo. Si anteriormente se indicaba que el uso de la tecnología, en cuanto a su uso por la sociedad, es excesivo, se deduce que hay conciencia del poder visual de la tecnología en el aula en detrimento de actividades táctiles y corporales, pero falta reflexión sobre el uso adecuado de la tecnología en el aula (ALONSO; GONZÁLVEZ; MUÑOZ, 2016), y en concreto en el ámbito infantil. La diversidad en el aula, por otro lado, está en relación directa a los recursos humanos. El profesorado percibe como muy positiva la inclusión de personas con diversidad funcional en el aula, ya que aumenta el sentimiento solidario del alumnado, aunque esta labor se complica enormemente si no es con apoyo de personal, en especial con alumnado que tiene comportamientos disruptivos. En esta línea son afines todas las investigaciones que potencian contextos inclusivos educativos, independientemente de que el comportamiento sea disruptivo o no (BOOTH; AINSCOW, 2015; FERNÁNDEZ, 2005/06; HOPKINS; AINSCOW; WEST, 1999; MORIÑA, 2004; PIJL; MEIJER; HEGARTY, 1997).

En cuanto a las categorías internas relativas a los recursos generales, del profesorado depende escuchar y prestar más atención a los intereses del alumnado, así como fomentar comunidad no sólo en el aula, sino fuera del aula.

Por último, las categorías internas relativas al cuerpo: el movimiento, la expresión corporal y la danza, así como la música y las técnicas de relajación. En cuanto a la música la valoración es muy positiva, se utiliza para tranquilizar al grupo, como también se comenta de las técnicas de relajación. Por otra parte, tanto el movimiento, la expresión corporal y la danza, como actividades que impliquen al cuerpo en general, no son una herramienta que se utilice en el aula, aunque se reconoce su importancia y la falta de formación en este campo, como lo subrayan autores anteriormente citados (LAGO; ESPEJO, 2007; MONTOYA, 2015; ORTÍZ, 2015). En confrontación con las experiencias en el ámbito corporal de Golding (2016) y Romero (2012), que destacan la especialización del docente y el aspecto cuantitativo del material dancístico, esta investigación hace hincapié en la accesibilidad de dicho material al docente no especializado en danza, otorgando una mejor conexión con su realidad educativa.

\section{CONCLUSIÓN}

Dando respuesta al objetivo planteado en un principio, las conclusiones de esta investigación determinan que las estrategias que utiliza el profesorado de los colegios implicados 
para optimizar la convivencia en el aula son la música y la visualización de imágenes o videos mediante pizarras digitales, obviando en cambio recursos relacionados con los aspectos corporales y sociales de una educación inclusiva. Reconoce su importancia y su efectividad, pero carece de motivación tanto para su aplicación en el aula como para formarse en este campo.

Por otro lado, le preocupa la tendencia individualista de la sociedad y manifiesta que se hace un uso excesivo de la tecnología fuera del aula. En cambio no es consciente de que un empleo inadecuado de la misma en el aula está potenciando la individualidad y está condicionando el trabajo cooperativo -reclama pizarras digitales sin plantearse las consecuencias de su utilización.

También le preocupa la elevada ratio de las aulas para atender adecuadamente al alumnado, y más cuando hay diversidad en las mismas, aunque halaga su valor como herramienta en el aula para mejorar la convivencia por el carácter solidario que imprime al grupo.

De todo esto se aprecia la necesidad de debate donde reflexionar sobre los efectos y el uso de la tecnología en el aula de infantil, así como de la verdadera presencia de diversidad en el aula.

Muchos de los problemas actuales de convivencia escolar existentes en la Comunidad andaluza no logran solucionarse con las tendencias actuales educativas, con lo que esta investigación puede despertar el interés del profesorado en aplicar nuevas líneas de acción. Por un lado, en el cuerpo, el movimiento y la danza como herramientas en una etapa educativa donde estas son esenciales para el desarrollo, y por otro lado, en la diversidad como contexto enriquecedor y solidario.

\section{REFERENCIAS}

AINSCOW, Mel; BOOTH, Tony. Guía para la Educación Inclusiva: Desarrollando el aprendizaje y la participación en los centros escolares. Madrid: Fuhem y OEI, 2015.

ALONSO, María Henar; GONZÁLVEZ, Juan Enrique; MUÑOZ, Ángel Bartolomé. Ventajas e inconvenientes del uso de dispositivos electrónicos en el aula: percepción de los estudiantes de grados en comunicación. Revista de Comunicación de la SEECI, v. 41, p. 136-154, 2016.

BANES, Sally. Terpsichore in sneakers: postmoderm dance. Middletown: Wesleyan University , 1987.

BENJAMIN, Adam. Making an Entrance: Theory and Practice for Disabled and Non-Disabled Dancers. New York: Routledge, 2002.

BERNSTEIN, Basil. Poder, educación y conciencia. Barcelona: El Roure, 1990.

BROZAS, María. Pedagogía del cuerpo sensible: tacto y visión en la danza Contact Improvisation. Movimento, v. 23, n. 3, p. 1039-1052, 2017.

BRUGAROLAS, Marisa. El cuerpo plural: Danza integrada en la inclusión. 2016. 751f.Tesis (Doctoral) - Universidad Politécnica de Valencia, 2016. 
CABERO, Julio. Reflexiones educativas sobre las tecnologías de la información y la comunicación. Tecnologia, Ciencia y Educación, n. 1, p. 19-27, 2015.

CASANOVA, M. ํㅡㄹ Antonia. La sociometría en el aula. Madrid: La Muralla, 1991.

DELEUZE, Gilles; GUATTARI, Félix. Mil mesetas. Valencia: Pre-textos, 2004.

FERNÁNDEZ, José María. ¿Educación inclusiva en nuestros centros educativos? Sí, pero ¿cómo? Contextos educativos. Revista de Educación, n. 8/ 9, p. 135-148, 2005-2006.

FERNÁNDEZ, Pablo; MELERO, Maa Ángeles. La interacción social en contextos educativos. Madrid: Siglo XXI de España , 1995.

FOUCAULT, Michel. Vigilar y castigar. Nacimiento de la prisión. Madrid: Editorial Biblioteca Nueva, 2012.

FREINET, Célestin. La formación de la infancia y de la juventud. Barcelona: Editorial Laia, 1972.

FROEBEL, Friedrich. La educación del hombre. Biblioteca Virtual Universal, 2003. Disponible en: <http://www.biblioteca.org.ar/libros/88736.pdfml>. Acceso: 9 sept. 2018.

GARCÉS, Marina. Un mundo común. Barcelona: Bellaterra, 2013.

GOBERNADO, Rafael. Individualismo y Colectivismo en el análisis sociológico. Revista Española de Investigaciones Sociológicas, n. 85, p. 9-25, 1999.

GOBERNADO, Rafael. La Comunidad: entre la integración y la igualdad. Revista Española de Investigaciones Sociológicas, n. 35, p. 113-121, 1986.

GOLDING, Alison; BOES, Claudia; NORDIN-BATES, Sanna. Investigating learning through developmental dance movement as a kinaesthetic tool in the Early Years Foundation stage. Research in Dance Education, v. 17, p. 1-33, 2016.

HENLEY, Matthew. Sensation, Perception, and Choice in the Dance Classroom. Journal of Dance Education, v. 14, n.3, p. 95-100, 2014.

HOPKINS, David; AINSCOW, Mel; WEST, Mel. School improvement in an era of change. London: Cassell, 1994.

HÜBNER, Fernanda. Contato improvisação (contact improvisation) um diálogo em dança. Movimento, v. 11, n. 2, p. 89-110, 2005.

KEMMIS, Stephen; McTAGGART, Robin. Cómo planificar la investigación acción. Barcelona: Alerter, 1988.

KEY, Ellen. El siglo de los niños. Barcelona: Henrich, 1906.

KIRK, Gordon. El currículum básico. Barcelona: Paidós-MEC, 1989.

KRAUSE, Mariane. Hacia una redefinición del concepto de comunidad. Revista de Psicología de la Universidad de Chile, v. 10, p. 49-60, 2001.

KUPPERS, Petra. Accesible Education: Aesthetics, bodies and disability. Research in Dance Education, v. 1, n.2, p. 119-131, 2000. 
LAGO, Pilar; ESPEJO, Alicia. El movimiento y la danza: su importancia dentro del currículo de Primaria. Educación y futuro: revista de investigación aplicada y experiencias educativas, n. 17, p. 149-164, 2007.

LE BRETON, David. Anthropologie du corps et modernité. París: Quadrige, 1990.

LLORENS, Juan Bautista. La diversidad en el aula de infantil a través de la danza integrada. Educación y Diversidad. Revista Inter-Universitaria de Investigación sobre Discapacidad e Interculturalidad, v. 10, n.1, p. 55-66, 2016.

LUZURIAGA, Lorenzo. Las nuevas escuelas. Madrid: Cosano, 1923.

MADRID, Dolores; MAYORGA, M. - José (Coords). La organización del espacio en el aula infantil. Barcelona: Octaedro, 2012.

MAKARENKO, Antón. Poema pedagógico. Barcelona: Planeta, 1977.

MARTINELLI, Flávia; DAOLIO, Jocimar; FILGUEIRA, Dulce. La cultura del cuerpo de los niños: diferencias y significados producidos en las clases de educación física. Movimento, v. 23, n. 4, p. 1217-1228, 2017.

MERLEAU-PONTY, Maurice. Fenomenología de la percepción. Barcelona: Planeta-De Agostini, 1993.

MONTESSORI, María. El método de la Pedagogía científica: Aplicado a la educación de la infancia. Madrid: Editorial Biblioteca Nueva, 2003.

MONTOYA, Juan Carlos. La formación musical de los futuros maestros de Educación Infantil y Primaria. La danza como dimensión indispensable. En: CHINCHILLA, Jose Luis; DÍAZ, Ana María (Coords.) Danza, Educación e Investigación. Málaga: Algibe, 2015. p. 47-53.

MORIÑA, Anabel. Teoría y Práctica de la Educación Inclusiva. Málaga: Aljibe, 2004.

NOVACK, Cynthia. Sharing the Dance: Contact Improvisation and American Culture. Madinson: University of Wisconsin, 1990.

ORTíz, María del Mar. La danza en la enseñanza obligatoria. En: CHINCHILLA, Jose Luis; DíAZ, Ana María (Coords.). Danza, Educación e Investigación. Málaga: Algibe, 2015. p. 15-34.

PALLASMAA, Juhani. The eyes of the skin: Architecture and the sense. Chichester: John Wiley, 2012.

PÉREZ GÓMEZ, Ángel. Educarse en la era digital: la escuela educativa. Madrid: Morata, 2012.

PIJL, Sip J.; MEIJER, Cor; HEGARTY, Seamus. Inclusive Education: A global agenda. Londres: Routledge, 1997.

ROBLIZO, Manuel; CÓZAR, Ramón. Usos y competencias en TIC en los futuros maestros de educación infantil y primaria: hacia una alfabetización tecnológica real para docentes. Pixel-Bit. Revista de Medios y Educación, n. 47, p. 23-39, 2015.

ROMERO, Javier. Percusión corporal y lateralidad. Método BAPNE. Revista Música y Educación, n. 91, p. 30-51, 2012. 
RUÍZ, M. a del Carmen; HERNÁNDEZ, Víctor Manuel. La incorporación y uso de las TIC en educación infantil. Un estudio de la infraestructura, la metodología didáctica y la formación del profesorado en Andalucia. Píxel-Bit. Revista de Medios y Educación, n. 52, p. 81-96, 2018.

RUSSELL, Joan. Creative Dance in the Primary School. Londres: McDonald \& Evans, 1965.

SPENCER, Paul. Society and the Dance. Cambridge: Cambridge University, 1985.

SUÁREZ, Magdalena. El grupo de discusión: Una herramienta para la investigación cualitativa. Barcelona: Laertes, 2005.

TAMPINI, Marina. Cuerpos e ideas en danza: Una mirada sobre el Contact Improvisation. Buenos Aires: Instituto Universitario Nacional del Arte, 2012. (Cuaderno de Danza n. 1).

TAYLOR, Steve J.; BOGDAN, Robert. Introducción a los métodos cualitativos de investigación. Argentina: Paidós, 1986.

TUDGE, Jonathan; ROGOFF, Barbara. Influencias entre iguales en el desarrollo cognitivo: perspectivas piagetiana y vygotskiana. En: FERNÁNDEZ, Pablo; MELERO, Maa Ángeles. La interacción social en contextos educativos. Madrid: Siglo XXI de España, 1995. p. 99-136.

Apoyo:

Universidad de Málaga 


\section{ANEXO 1: UNIDAD DIDÁCTICA SAMBA LELÉ}

A continuación se muestra la forma en la que los aspectos corporales y sociales de una educación inclusiva se evidencian en una unidad didáctica. En primer lugar se relacionan en un cuadro dichos aspectos con los objetivos del programa educativo:

Cuadro 2 - Relación de los aspectos corporales y sociales con el programa educativo

\begin{tabular}{|l|l|c|}
\hline $\begin{array}{l}\text { Aspectos } \\
\text { corporales y } \\
\text { sociales }\end{array}$ & Objetivos del programa educativo & Objetivo no \\
\hline \multirow{2}{*}{ Exploración } & $\begin{array}{l}\text { Explorar nuestras possibilidades de movimientos mediante propuestas } \\
\text { abiertas o semiabertas corporales. }\end{array}$ & 1 \\
\hline \multirow{5}{*}{ Sentido del tacto } & $\begin{array}{l}\text { Desarrollar el sentido del tacto individualmente y con el "otro": juegos } \\
\text { de contacto, percusión corporal, movimiento y danza. }\end{array}$ & 2 \\
\cline { 2 - 3 } & Comunicarmos con el "otro" desde el cuerpo y el sentido del tacto. & 3 \\
\hline \multirow{5}{*}{ Comunitarismo } & $\begin{array}{l}\text { Desarrollar el sentido del tacto individualmente y con el "otro": juegos } \\
\text { de contacto, percusión corporal, movimiento y danza. }\end{array}$ & 2 \\
\cline { 2 - 3 } & Comunicarmos con el "otro" desde el cuerpo y el sentido del tacto. & 3 \\
\cline { 2 - 3 } & $\begin{array}{l}\text { Potenciar la cultura tradicional a través de las canciones y danzas } \\
\text { populares. }\end{array}$ & 4 \\
\cline { 2 - 3 } & $\begin{array}{l}\text { Conocer las diferentes culturas del mundo a través de las canciones } \\
\text { del mundo. }\end{array}$ & 5 \\
\hline
\end{tabular}

Fonte: Elaboración propia

Hay que aclarar que cuando se refiere a desarrollar el sentido del tacto, tanto individualmente como con el "otro", no se hace referencia a un desarrollo manipulativo táctil, sino a una concepción más amplia donde el tacto se experimenta desde toda la piel.

En segundo lugar se vinculan los objetivos del programa educativo con las diferentes fases del procedimiento de la unidad didáctica.

Cuadro 3 - Relación de los objetivos del programa educativo con el procedimiento de la unidad

\begin{tabular}{|l|l|l|}
\hline Objetivos & Procedimiento & Actividad \\
\hline 1 & Introdución & Canción A todos nos gusta saludar - Calentamiento corporal \\
\hline $1,2,5$ & Presentación & Narración ¿A qué sabe la luna? - Canción y coero Samba lelé \\
\hline $1,2,3$ & Exploración & Danza de palos - Canción Samba lelé \\
\hline $1,2,3,5$ & Actividad principal & Canción y coero Samba lelé, y danza de palos \\
\hline 1 & Relajación & Respiración \\
\hline & & Fonte: Elaboración propia
\end{tabular}

En cuanto al procedimiento a seguir, se comienza con la introducción, un calentamiento corporal utilizando la canción $A$ todos nos gusta saludar (véase anexo 2), en la que se invita a mover libremente diferentes partes del cuerpo a la vez que se saluda en el idioma original de la canción Samba lelé diciendo "olá". Se sigue con la presentación de la temática, una pequeña narración basada en el cuento de Michael Grejniec ¿A qué sabe la luna?. En ella se 
introduce una pequeña coreografía a modo de rondó musical y de danza con la canción Samba lelé (véase anexo 2). El cuento adaptado es el siguiente: "Érase una vez un gran bosque en Brasil, donde vivían muchos animales que querían saber cómo sabía la luna. Aparece la tortuga (utilizar objetos amontonables con las imágenes de los animales) subiendo a la cima de la montaña más alta (cantar y bailar el estribillo). Al llegar a la cima no alcanza la luna, y decide llamar al elefante: elefanteeeee... El elefante sube a la cima de la montaña cantando la canción (cantar y bailar el estribillo). Al llegar a la cima se sube encima de la tortuga, y tampoco alcanza la luna. Entonces deciden llamar a la jirafa (repetir igualmente con otros animales). Al final el ratón consigue alcanzar la luna, coge un trozo, la prueba y la reparte entre todos los animales".

En la exploración se hace un juego de equilibrio con palos de cartón inspirado en la danza de palos brasileña de origen afro-brasileño Maculelé. El profesor canta la canción Samba lelé mientras el alumnado juega en parejas a mantener en equilibrio el palo. Cada uno de ellos pone un extremo del palo en la palma de su mano.

En la actividad principal todos cantan y bailan la coreografía en la estrofa, y en el estribillo bailan en parejas manteniendo en equilibrio el palo. La coreografía puede ser diseñada por el profesor o por el grupo.

Por último, un momento de relajación, donde se presta atención a la respiración mientras que el profesor toca o canta alguna melodía. 


\section{ANEXO 2: CANCIONES Y PARTITURAS}

Canción $\boldsymbol{A}$ todos nos gusta saludar (adaptación de la canción Ich grüsse alle). Canción de saludo compuesta por el músico alemán Volker Rosin. Letra adaptada al inglés por Shirley Salmon, y al español por Juan Bautista Llorens. En la segunda parte se han introducido partes del cuerpo como ejercicio de calentamiento y de conciencia corporal.

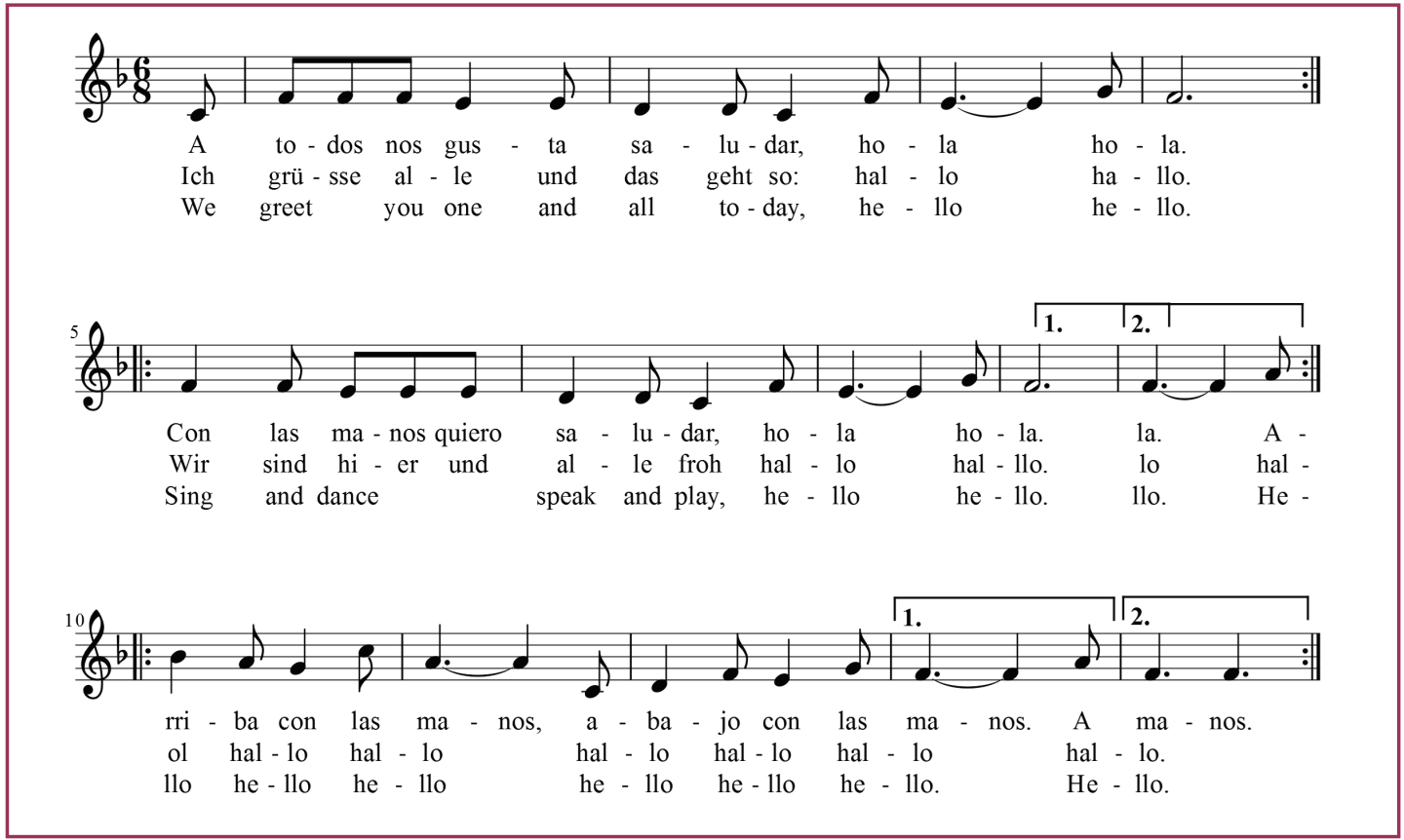

Canción popular brasileña Samba lelé. Versión adaptada al español por Juan Bautista Llorens.
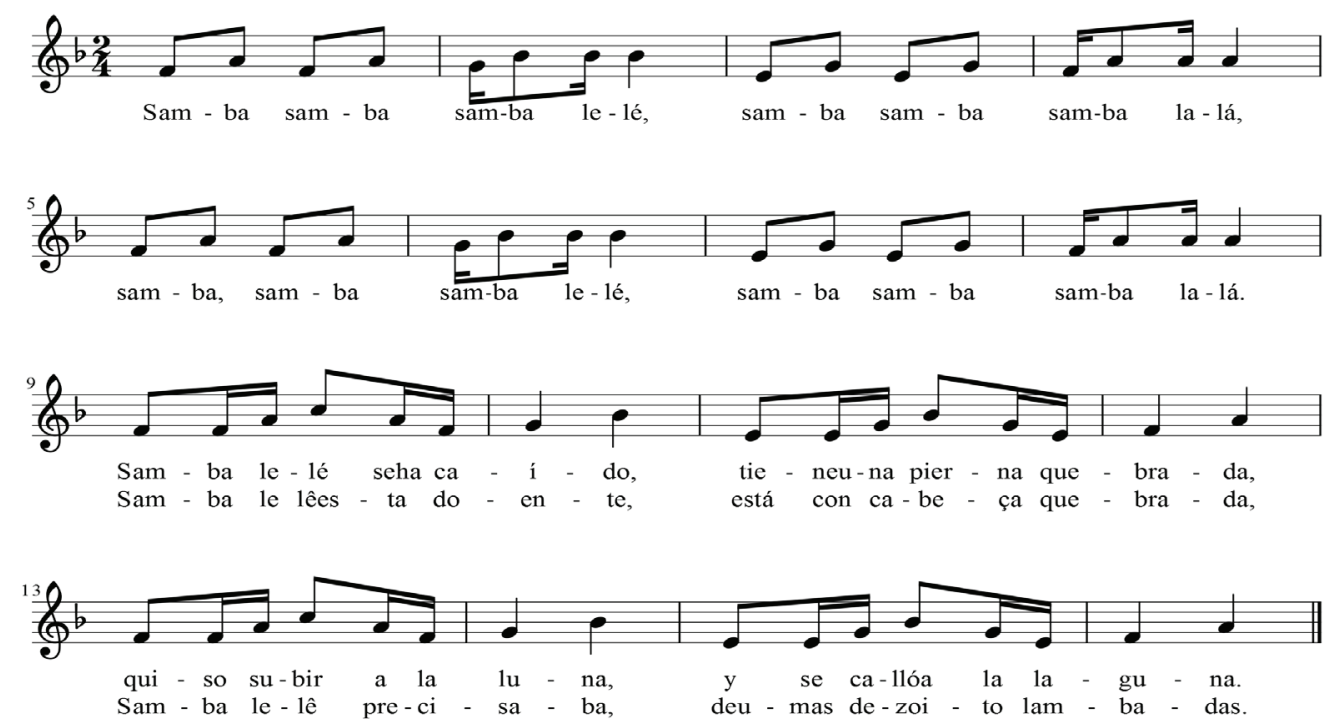